\title{
Evidence for the Regulation of Actin-myosin Binding Strength by Lever Arm and Subfragment-2 Regions of Myosin Molecule in Contracting Skinned Muscle Fibers as Revealed by the Effect of Antibodies
}

\section{Sugi $\mathbf{H}^{1 *}$ and Chaen $\mathbf{S}^{2}$}

${ }^{1}$ Department of Physiology, School of Medicine, Teikyo University, Tokyo, Japan

${ }^{2}$ Department of Biosciences, College of Humanities and Sciences, Nihon University, Tokyo, Japan

\begin{abstract}
It is generally believed that myosin heads $(M)$ extending from myosin filaments form weak binding with actin filament (A) in the form of A-M-ADP-Pi, and strong binding with $\mathrm{A}$ in the form of A-M-ADP and A-M. During muscle contraction, $M$ first attaches weakly to $A$ in the form of M-ADP-Pi, and then release $P i$ to attach strongly with $A$ in the form of $A-M-$ ADP to exert power stroke producing myofilament sliding. The weak to strong binding transition between $M$ and $A$ is therefore essential for producing muscle contraction. After power stroke, associated with release of ADP, M detaches from A on binding with next ATP to form M-ADP-Pi. Thus, M repeats attachment-detachment cycle with A, coupled with ATP hydrolysis. Using antibodies to myosin lever arm domain (anti-LD antibody) and to myosin subfragment-2 region (anti-S-2 antibody), we have found that, if these antibodies attach to LD or S-2 regions, myosin heads no longer generate force, but still hydrolyse ATP at a normal rate without contributing to muscle fiber stiffness and internal resistance against fiber shortening. The puzzling effect of antibodies can be accounted for by assuming that in myosin molecules, with antibodies attached to their LD or S-2 region, (1) the strength of A-M binding is markedly reduced to be readily broken by small length perturbations or muscle fiber shortening, and (2) such myosin heads still repeat attachmentdetachment cycle coupled with ATP hydrolysis without passing through weak to strong A-M binding transition. Thus, the present work indicates functional influence of LD and S-2 regions on the A-M binding of myosin molecule, suggesting essential role of these regions in muscle contraction.
\end{abstract}

Keywords: Muscle contraction; Actin-myosin interaction; Weak to strong actin-myosin binding transition; Skinned muscle fiber; Anti-LD antibody; Anti-S-2 antibody

\section{Structure of Myofilament Lattice in Muscle and the Sliding Filament Mechanism in Muscle Contraction}

Muscle contraction results from cyclic attachment-detachment between myosin heads extending from myosin filaments and the corresponding sites on actin filaments, coupled with ATP hydrolysis $[1,2]$. As illustrated in Figure 1A, a myosin molecule (MW, 450,000) has two pear-shaped heads and a rod of $156 \mathrm{~nm}$ long, and can be split into two parts: (1) the rod of $113 \mathrm{~nm}$ long, known as light meromyosin (LMM), and (2) the rest of the myosin molecule, containing the two heads and a rod of $43 \mathrm{~nm}$ long, known as heavy meromyosin (HMM). The HMM can be further split into two separate heads, i.e. subfragment-1 (S-1), and a rod, i.e. subfragment-2 (S-2). In myosin filaments, LMM aggregates to form filament backbone, which is polarized in opposite directions across the myosin filament central region, called bare region (Figure 1B). The S-2 rod serves as a hinge betwwn the S-1 head and the filament backbone, thus enabling the S-1 head to swing away from the filament. The axial separation of the two $\mathrm{S}-1$ head in myosin filament is $14.3 \mathrm{~nm}$.

Actin filaments consists primarily of two helical strands of globular actin monomers (MW, 41,700), which are wound around each other with a pitch of $35.5 \mathrm{~nm}$. The axial separation of actin monomers in actin filament is $5.46 \mathrm{~nm}$ (Figure 1C). In vertebrae skeletal muscle, actin filaments also contain two regulatory proteins, tropomyosin and troponin. In relaxed muscle, interaction between S-1 heads and actin monomers are inhibited by tropomyosin. When $\mathrm{Ca}^{2+}$ binds to troponin, it removes the inhibitory effect of tropomyosin to start actin-myosin interaction, i.e. muscle contraction. In skinned muscle fibers, from which surface membrane is removed by treatment with glycerol, the fibers can be activated to contract by external application of $\mathrm{Ca}^{2+}$. Figure 1D shows longitudinal arrangement of actin and myosin filaments in muscle. Actin filaments extend in either direction from the Z-line, and penetrate in between myosin filaments, which are located central in each sarcomere, i.e. the structure between adjacent $Z$-lines to serve as structural and functional unit of muscle. In each sarcomere, the region containing only actin filaments is called the I-band, while the region containing both actin and myosin filaments are called the A-band. It has been well established that, when a muscle is activated to contract, relative sliding between actin and myosin filaments takes place, in such a way that actin filaments are further pulled into myosin filament arrays, while the length of actin and myosin filaments remain constant $[3,4]$. After the monumental discovery of the sliding filament mechanism, the central problem in the research field of muscle contraction is: what makes actin and myosin filaments slide past each other? Both the ATPase activity and the actin binding sites are localized in S-1 heads, which will hereafter be called myosin heads. Consequently, it is generally agreed that myosin heads, extending from myosin filaments towards actin filaments, play a major role in the myofilament sliding.

*Corresponding author: Sugi $\mathrm{H}$, Department of Physiology, School of Medicine Teikyo University, Tokyo, Japan, Tel: +81484784079; E-mail: sugi@kyf.biglobe.ne.jp

Received December 21, 2016; Accepted December 27, 2016; Published December 31, 2016

Citation: Sugi H, Chaen S (2016) Evidence for the Regulation of Actin-myosin Binding Strength by Lever Arm and Subfragment-2 Regions of Myosin Molecule in Contracting Skinned Muscle Fibers as Revealed by the Effect of Antibodies. J Nanomed Nanotechnol 7: 415. doi: 10.4172/2157-7439.1000415

Copyright: (c) 2016 Sugi $\mathrm{H}$, et al. This is an open-access article distributed under the terms of the Creative Commons Attribution License, which permits unrestricted use, distribution, and reproduction in any medium, provided the original author and source are credited. 
Citation: Sugi H, Chaen S (2016) Evidence for the Regulation of Actin-myosin Binding Strength by Lever Arm and Subfragment-2 Regions of Myosin Molecule in Contracting Skinned Muscle Fibers as Revealed by the Effect of Antibodies. J Nanomed Nanotechnol 7: 415. doi: 10.4172/21577439.1000415

Page 2 of 8

A

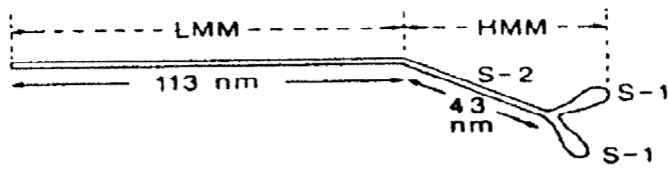

B

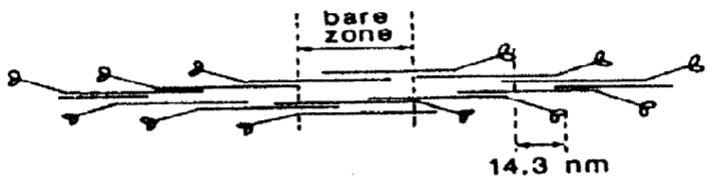

C

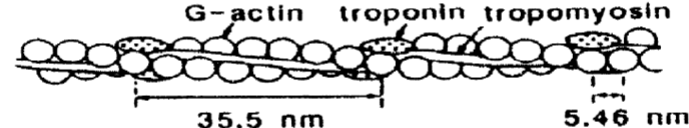

$\mathrm{D}$

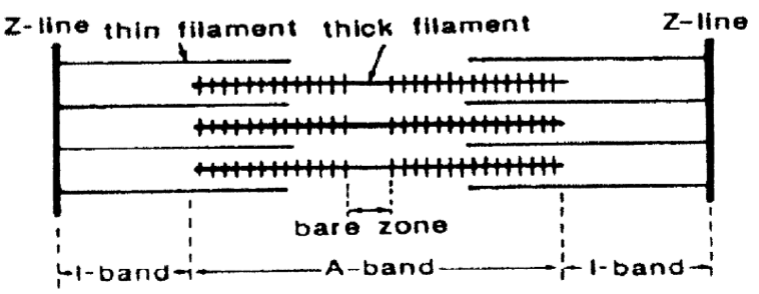

Figure 1: Structure of myosin molecule (A). myosin filament (B). actin filament (C). and arrangement of myofilaments within a sarcomere (D). For further explanation, see text [1].

\section{Attachment-Detachment Cycle between Myosin Heads and Actin Filaments}

Figure 2 illustrates the idea that occurred to H.E. Huxley when he discovered the sliding filament mechanism, in which actin and myosin filaments slide past each other without changing their length [5]. Due to the difference in periodicity between myosin heads extending from myosin filaments and the myosin-binding sites on actin filaments, the interaction between myosin heads and actin filaments takes place asynchronously. In the upper diagram, a myosin head (located on the left) extending from myosin filament first attaches to a myosin-binding site on actin filament. Then, the attached head changes its configuration to produce a unitary sliding between actin and myosin filaments (middle diagram); the direction of sliding is such that the length of sarcomere shortens as actin filament moves into myosin filament arrays, i.e. the A-band. After producing the unitary filament sliding, myosin head detaches from actin filament (lower diagram). It is generally believed that hydrolysis of one ATP molecule is coupled with each cycle of actinmyosin interaction shown in Figure 2.

Despite extensive studies, including experiments on muscle mechanics, time-resolved X-ray diffraction, chemical probes, and cryo-electron microscopy, no definite conclusion has been reached with respect to changes in configuration of myosin heads coupled with ATP hydrolysis. Recently, however, our research group have succeeded in visualizing and recording ATP-induced movements in individual myosin heads in hydrated myosin filaments [6-9], though much more experimental work is necessary for full understanding of muscle contraction at the molecular level.

\section{Biochemical Reaction Steps of Actomyosin ATPase Related to Muscle Contraction}

Muscle is regarded as a machine converting chemical energy derived from ATP hydrolysis into mechanical work. The ATPase activity of myosin, HMM or S-1 is very slow, but increases markedly in the presence of actin filaments (F-actin). This phenomenon is called actin-activation of myosin ATPase activity, and has been studied mainly by the quenched flow or the stopped flow method, in which myosin sample is rapidly mixed with actin filament, to measure release of ATP hydrolysis products, i.e. inorganic phosphate (Pi) and ADP [2]. It should be noted, however, that, to perform biochemical experiments in water solution, myosin (or HMM and S-1) concentration is kept to be a few micromolar, compared to the myosin head concentration in muscle $(100-200 \mu \mathrm{M})$, and the $3 \mathrm{D}$ myofilament lattice structure is completely lost. Despite the above marked difference between actomyosin (mixture of actin filament and myosin, HMM or S-1) solution and myofilament lattice in muscle, the most plausible sequence of reaction occurring in contracting muscle, known as the Lymn-Taylor scheme, has been presented (Figure 3) [10].

In this scheme, myosin heads $(\mathrm{M})$ in relaxed muscle are in the form of M-ADP-Pi, and hydrolyse ATP at an extremely slow rate. On muscle activation, $\mathrm{M}$ attaches to actin filament (A) to form complex A-M$\mathrm{ADP}-\mathrm{Pi}$, which releases $\mathrm{Pi}$ and ADP with a much faster rate as a result of actin-activation of myosin ATPase activity. Based on measurement of equilibrium dissociation constants in solution, it is generally believed that M-ATP and M-ADP-Pi have a low affinity for actin, while M-ADP and $\mathrm{M}$ alone have a high affinity for actin [2]. These results indicate the following sequential reactions, which are associated with myosin head power stroke, producing muscle contraction: (1) M-ADP-Pi $\rightarrow$ A-M-ADP-Pi, i.e. weak A-M binding; (2) A-M-ADP-Pi $\rightarrow$ A-M-ADP $+\mathrm{Pi}$, i.e. weak to strong A-M binding transition; (3) A-M-ADP $\rightarrow$ A-M $+\mathrm{ADP}$, i.e. myosin head power stroke, associated with conformational

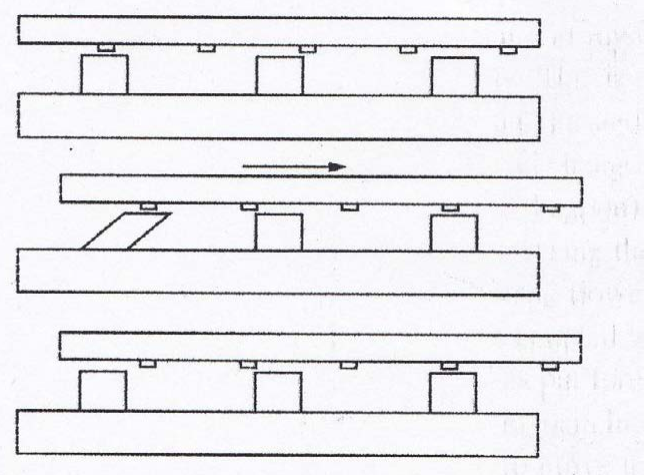

Figure 2: Proposed attachment-detachment cycle between myosin heads extending from myosin filament and corresponding myosin-binding sites on actin filament [5].

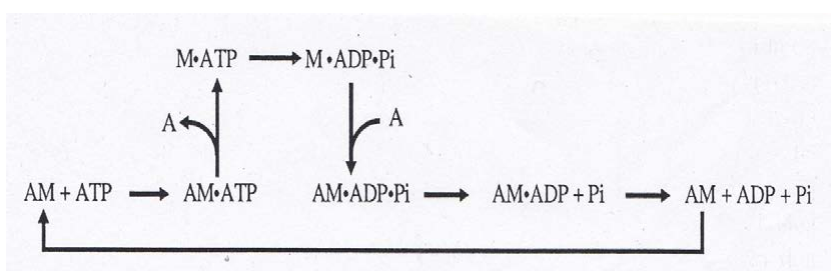

Figure 3: Lymn-Taylor scheme of cyclic reaction steps. In each cycle, one ATP molecule I hydrolysed into ADP and $\mathrm{Pi}$. M and $\mathrm{A}$ denote myosin head and actin filament, respectively. 
Citation: Sugi H, Chaen S (2016) Evidence for the Regulation of Actin-myosin Binding Strength by Lever Arm and Subfragment-2 Regions of Myosin Molecule in Contracting Skinned Muscle Fibers as Revealed by the Effect of Antibodies. J Nanomed Nanotechnol 7: 415. doi: 10.4172/21577439.1000415

Page 3 of 8

changes of myosin heads while attached strongly to actin filaments; (4) $\mathrm{A}-\mathrm{M}+\mathrm{ATP} \rightarrow \mathrm{M}-\mathrm{ATP}+\mathrm{A}$, i.e. detachment of $\mathrm{M}$ from A upon binding with next ATP, and (5) M-ATP $\rightarrow$ M-ADP-Pi, i.e. myosin head recovery stroke, associated with ATP hydrolysis.

The above sequence of events can be illustrated in a realistic manner in Figure 4. This putative scheme of myosin head power and recovery strokes are now visualized and recorded by our research group using the gas environmental chamber (EC); the magnitude of myosin head recovery stroke under practically unloaded condition is $\sim 7 \mathrm{~nm}[7,9]$. In contracting muscle, the attachment-detachment cycle between $\mathrm{M}$ and A, each coupled with hydrolysis of one ATP molecule is going on rapidly with actin-activated ATPase activity. According to the biochemical scheme shown in Figures 3 and 4, the ATPase activity of contracting muscle is tightly coupled with weak to strong transition (and also with attachment-detachment cycle) of A-M binding.

\section{Muscle Fiber Stiffness as a Measure of Myosin Heads Strongly Attached to Actin Filaments}

Since the strong A-M binding may not break with small applied displacements, muscle fiber stiffness, serving as a measure of strong A-M binding, is widely measured by applying small sinusoidal vibrations (amplitude, $\sim 0.1 \%$ of slack fiber length, Lo; frequency $\mathrm{kHz}$ region) and recording resulting force changes. Muscle fiber stiffness provides information about what takes place in contracting muscle. During the development of isometric force, muscle fiber stiffness rises ahead of force [11], indicating that, when strong A-M bindings are formed, a finite time is required for them to develop isometric force (Figure 5A). Muscle fiber stiffness during steady isotonic shortening has been measured under various isotonic loads (=force), and is found to decrease to a finite minimum value with decreasing isotonic load from the maximum value Po to zero [12] (Figure 5B), being consistent with the contraction model of Huxley [13].

In the Huxley contraction model, it was assumed that both actin and myosin filaments were completely rigid, so that the measured stiffness originated only from the compliance of strong A-M bindings. By stretching rigor muscle fibers and observing the resulting changes in sarcomere structures electron microscopically, however, Suzuki and Sugi [14] have demonstrated that both actin and myosin filaments

A
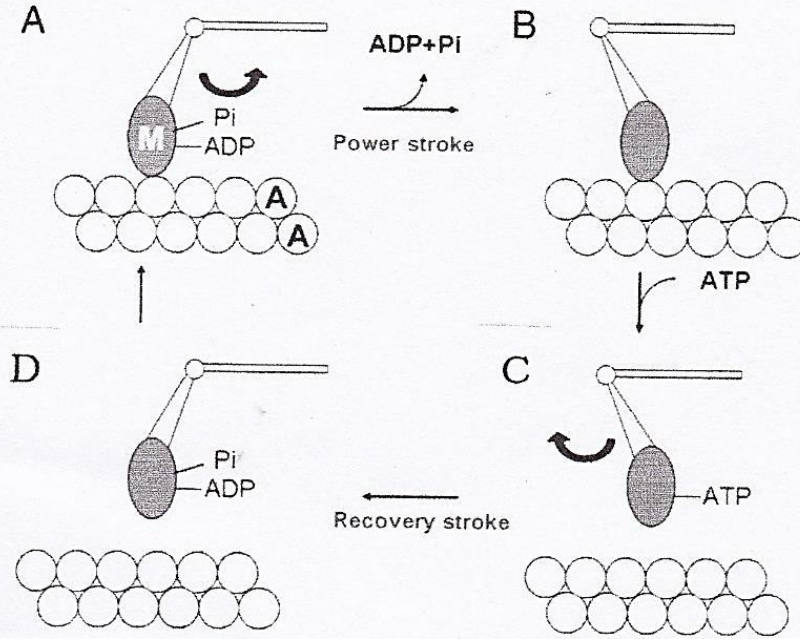

Figure 4: Diagrammatic illustration of Lymu-Taylor cyclic reaction steps, in which myosin head $(\mathrm{M})$ repeats attachment to, and detachment from, actin filament (A), coupled with power and recovery strokes, respectively [7]. are extensible, indicating that a considerable fraction of muscle fiber stiffness originates from myofilament compliance (Figure 6). This was later confirmed by time-resolved X-ray diffraction studies on contracting muscle [15]. Despite these results, muscle fiber stiffness still remains to be a valid measure of fraction of myosin heads strongly bound to actin filaments at any one moment.

\section{Effect of p-Phenylene Dimaleimide on Contraction Characteristics and MgATPase Activity in Single Skinned Muscle Fibers}

A bifunctional thiol reagent, p-phenylenedimaleimide (p-PDM) is known to react with reactive sulphydrils on the distal region of myosin head to result in loss of its ability to bind with actin filament and to hydrolyse MgATP, the substrate of myosin and actomyosin ATPase [16]. On the other hand, p-PDM has no effect on actin filament binding to myosin head [17]. We examined the effect of p-PDM on contraction characteristics and MgATPase activity in single $\mathrm{Ca}^{2+}$-activated skinned muscle fibers [18]. Partially p-PDM- treated muscle fibers contain mixture of myosin molecules with zero, one or two of their heads inactivated with p-PDM $[16,19]$. Based on the assumption that two heads of each myosin molecules react with p-PDM independently from each other, the three species of myosin molecules within the fiber can be estimated from simple probabilistic calculations. As illustrated in Figure 7, the fraction of two-head-inactivated myosin molecules is proportional to the square of the total fraction of myosin molecules reacted with p-PDM.
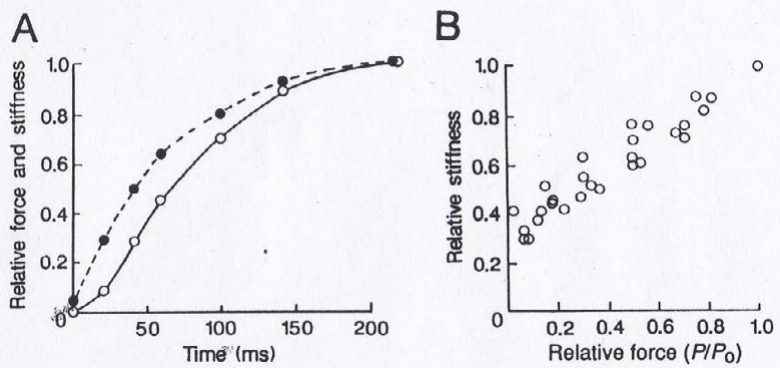

Figure 5: Muscle fiber stiffness widely used as a measure of number of myosin head performing power and recovery strokes, coupled with ATP hydrolysis, during contraction. (A) Changes in force (open circles) and stiffness (filled circles) during the rise of isometric force in a single skeletal muscle fiber [11] (B) Relation between stiffness and isotonic load (=force) during steady isotonic shortening of a single skeletal muscle fiber [12].

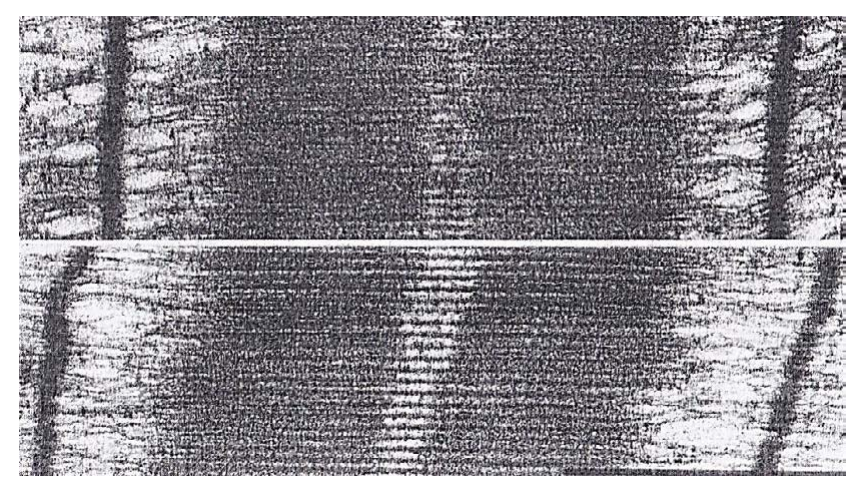

Figure 6: Electron micrographs showing marked extensibility of actin and myosin filaments, revealed by comparing sarcomere structure before (A) and after (B) stretch of a single skeletal muscle fiber in rigor state. Calibration bar at the bottom, $1 \mu \mathrm{m}[13]$. 
Citation: Sugi H, Chaen S (2016) Evidence for the Regulation of Actin-myosin Binding Strength by Lever Arm and Subfragment-2 Regions of Myosin Molecule in Contracting Skinned Muscle Fibers as Revealed by the Effect of Antibodies. J Nanomed Nanotechnol 7: 415. doi: 10.4172/21577439.1000415

Page 4 of 8

The Magnitude of $\mathrm{Ca}^{2+}$-activated isometric force in partially p-PDMtreated fibers, expressed relative to the control value in untreated fibers as $\mathrm{T} / \mathrm{To}$, was found to be proportional to the square of the MgATPase activity of p-PDM-treated fibers, also expressed relative to the control value as $\mathrm{J} / \mathrm{Jo}$, indicating that the MgATPase activity in $\mathrm{p}$-PDM-treated fibers is proportional to the total fraction of native myosin heads (1-q), while the isometric force is proportional to the value $(1-q)^{2}$; namely, isometric force is generated only by myosin molecules with two native heads, while all native myosin heads hydrolyse ATP at a similar rate independently of each other (Figure 8).

In p-PDM untreated control fibers, muscle fiber stiffness, expressed relative to the control value $\mathrm{S} / \mathrm{So}$ increased linearly with increasing $\mathrm{Ca}^{2+}$ activated isometric force (crosses, Figure 9, left). Meanwhile, in p-PDM treated fibers, the stiffness, expressed as S/So, exhibited a curved T/To versus S/So relation (filled circles, Figure 9, left), indicating that the stiffness is proportional to the total fraction of native myosin heads (1q). These results show that all native heads hydrolyse ATP at a similar rate, irrespective of whether the other myosin head in the same myosin molecule is inactivate with p-PDM or remains native; in other words, native myosin heads in myosin molecules, with one of their heads inactivated with p-PDM, can no longer participate in isometric force generation, but still repeat detachment-attachment cycle with actin filaments. The above explanation is consistent with the idea that the stiffness serves as a measure of total number of myosin heads repeating attachment-detachment cycle with actin, coupled with ATP hydrolysis,

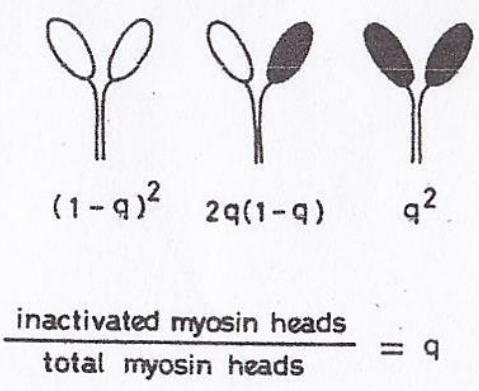

Figure 7: Three different species of myosin molecules and their respective fractions calculated on the assumption of independent reaction of myosin two heads with $\mathrm{p}$-PDM. Open and slid heads represent native and p-PDMinactivated heads, respectively [17].

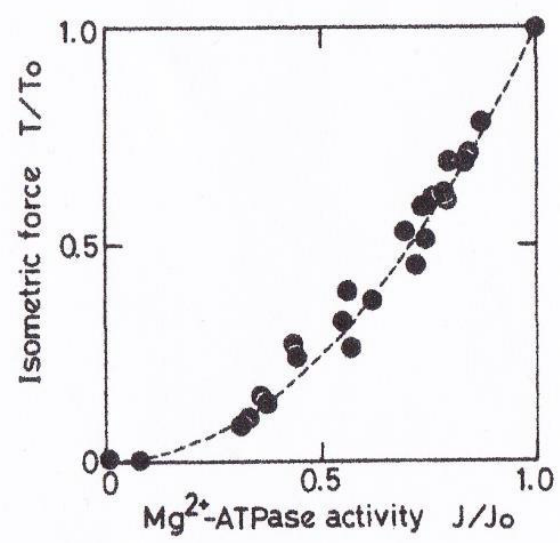

Figure 8: Relation between $\mathrm{Ca}^{2+}$-activated isometric force $(\mathrm{T})$ and MgATPase activity $(\mathrm{J})$ in p-PDM-treated single skinned skeletal muscle fibers. Both $\mathrm{T}$ and $\mathrm{J}$ are expressed relative to control values, To and Jo, respectively [17]. irrespective of whether they generate force or not. Since the non forcegenerating myosin heads, still hydrolyse ATP by repeating transition from weak to strong A-M binding, they are expected to produce appreciable internal resistance against muscle fiber shortening. In fact, the maximum unloaded shortening velocity of p-PDM treated fibers, as measured by the slack test [19], and expressed relative to the control value $\mathrm{V} / \mathrm{Vo}$, was found to decrease with decreasing isometric force $\mathrm{T} /$ To (Figure 9, right).

To summarize, if one of the two heads of a myosin molecule is inactivated with p-PDM, the remaining one native head (1) still hydrolyses ATP at a rate similar to the control value, (2) is sensed as stiffness measured in response to small length perturbation, and (3) produces appreciable internal resistance against fiber shortening. These results are consistent with the dogma that myosin heads generate force in muscle fibers by repeating attachment-detachment cycle, associated with weak to strong A-M binding transition. This dogma does not hold, however, when antibodies are attached to myosin head LD or myosin $\mathrm{S}-2$, as will be described in the following sections.

\section{Effect of Anti-S-2 Antibody on Contraction Characteristics and MgATPase Activity in Single Skinned Muscle Fibers}

In 1992, we were asked by Dr. Harrington to examine the effect of polyclonal antibody to myosin subfragment-2 (S-2), prepared in his laboratory. As illustrated in Figure 1, the S-2 connects myosin head to myosin filament backbone. The anti-S-2 antibody was confirmed electron microscopically to attach specifically to the S-2 region of myosin molecule (Katayama, unpublished observation). In the presence of anti-S-2 antibody (up to $1.5 \mathrm{mg} / \mathrm{ml}$ ), the magnitude of $\mathrm{Ca}^{2+}$-activated isometric force gradually decreased with time, while the MgATPase activity did not change appreciably even when the isometric force was reduced to zero (Figure 10), indicating that myosin molecules, with the antibody attached to their S-2 region, no longer generate isometric force, while still hydrolyzing ATP at a normal rate. Meanwhile, in anti-S-2 antibody-treated fibers, muscle fiber stiffness decreased in proportion to decreasing isometric force (Figure 11), indicating that the stiffness also serves as a measure of force-producing myosin molecules within the fiber.
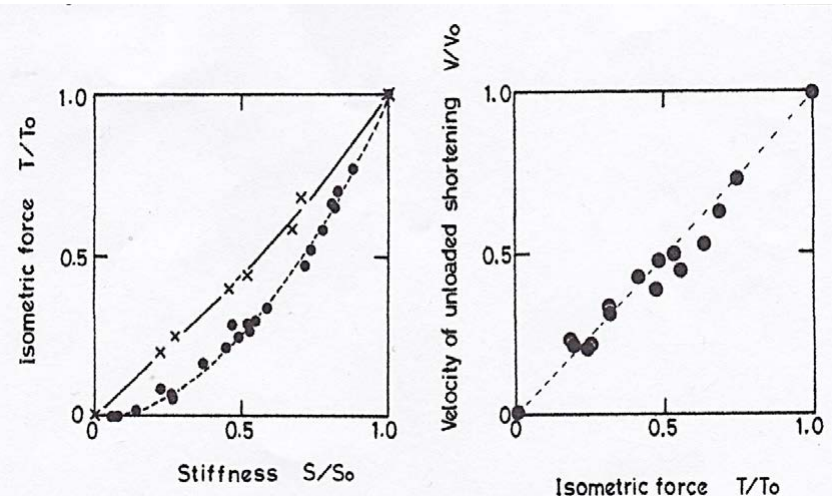

Figure 9: (Left) Relation between $\mathrm{Ca}^{2+}$-activated isometric force $(\mathrm{T})$ and muscle fiber stiffness (S) in p-PDM-treated (filled circles) and untreated control fibers (crosses). Both $\mathrm{T}$ and $\mathrm{S}$ are expressed relative to control values, To and So, respectively. Broken line shows the curve calculated from equation, $(\mathrm{T} / \mathrm{To})=(\mathrm{S} /$ So $)^{2}$. (Right) Relation between $\mathrm{Ca}^{2+}$-activated isometric force $(\mathrm{T})$ and maximum unloaded velocity of shortening $(\mathrm{V})$ in $\mathrm{p}$-PDM treated fibers. Both $\mathrm{T}$ and $\mathrm{V}$ are expressed relative to control values, To and Vo, respectively [17]. 
Citation: Sugi H, Chaen S (2016) Evidence for the Regulation of Actin-myosin Binding Strength by Lever Arm and Subfragment-2 Regions of Myosin Molecule in Contracting Skinned Muscle Fibers as Revealed by the Effect of Antibodies. J Nanomed Nanotechnol 7: 415. doi: 10.4172/21577439.1000415

Page 5 of 8

A

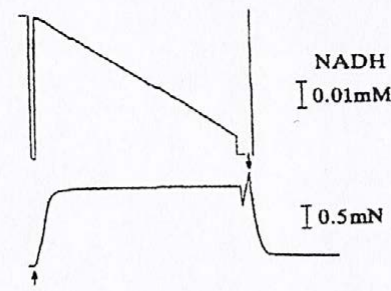

B

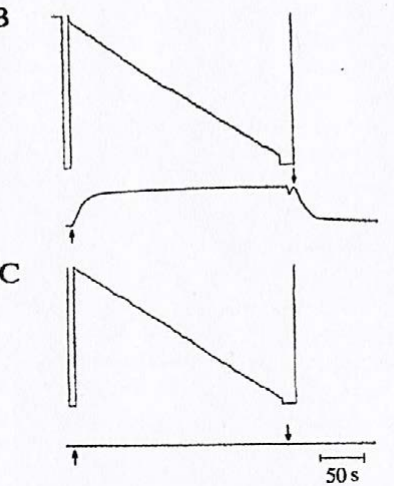

(=loads) by one shot (Figure 12) [22]. Unexpectedly, the maximum unloaded velocity of shortening Vmax in antibody-treated fibers, did not change appreciably despite the marked reduction of isometric force (Figure 13A); if the force-velocity curves were normalized with respect to the maximum force, the curves were found to be identical (Figure 13B). This unexpected finding provides a puzzling question, why myosin heads, with antibodies attached to their S-2 region, can still hydrolyse ATP at a normal rate without producing any appreciable internal resistance against fiber shortening and without being sensed as the stiffness by small length perturbations? The same puzzling result is obtained with respect to the effect of antibody to myosin head lever arm domain (LD), located between myosin S-1 head and myosin S-2 regions.

A

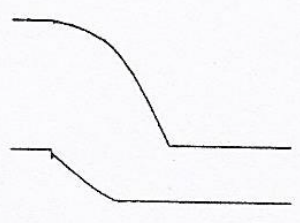

C

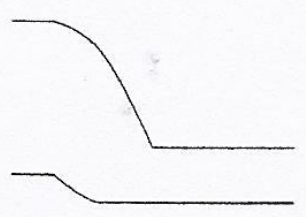

B

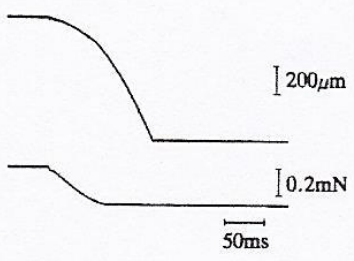

$\mathrm{D}$

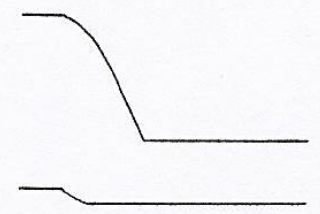

Figure 12: Fiber length (upper traces) and isometric force (lower traces) records in response to ramp decreases in force in a single skinned muscle fiber before (A9 and after 30 (B), 60 (C) and $90 \mathrm{~min}$ (D) after application of anti-S-2 antibody $(1.5 \mathrm{mg} / \mathrm{ml})[21]$.

A

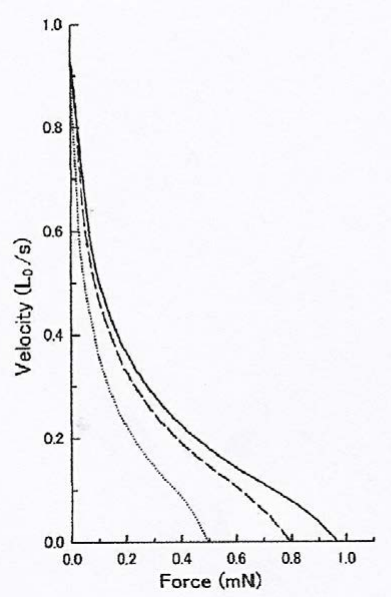

B

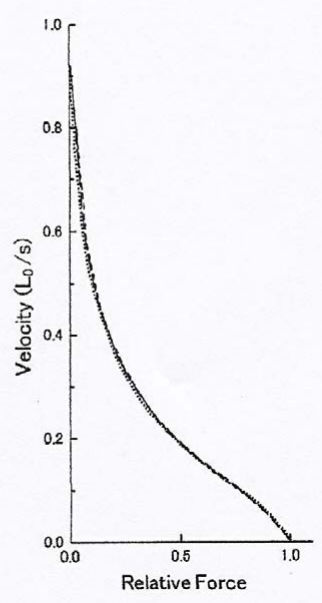

Figure 11: Relation between muscle fiber stiffness and $\mathrm{Ca}^{2+}$-activated isometric force in a single skinned muscle fiber before (curve A) and 30 (curve B), 60 (curve C) and 90 min (curve D) after application of anti-S-2 antibody (1.5 mg/ $\mathrm{ml}$ ). Both isometric force and stiffness are expressed relative to control values, respectively [21].

We also examined the force-velocity $(\mathrm{P}-\mathrm{V})$ relation in control and antibody-treated fibers. In chemically skinned fibers, it is difficult to obtain a number of data points enough to obtain $\mathrm{P}-\mathrm{V}$ relation due to gradual deterioration at the two cut ends [20,21]. To overcome this difficulty, we constructed a force-clamp circuit, with which ramp decreases in force can be applied to the fiber at various levels of steady isometric force in both control and antibody-treated fibers, so that we could obtain continuous $\mathrm{P}-\mathrm{V}$ relation over the whole range of forces

Figure 13: (A) Force-velocity curves of a single skinned muscle fiber before (solid line) and after application of anti-S-2 antibody $(1.5 \mathrm{mg} / \mathrm{ml}$ ) (broken and dotted lines). (B) The same force-velocity curves normalized with respect to maximum isometric forces [21]. 
Citation: Sugi H, Chaen S (2016) Evidence for the Regulation of Actin-myosin Binding Strength by Lever Arm and Subfragment-2 Regions of Myosin Molecule in Contracting Skinned Muscle Fibers as Revealed by the Effect of Antibodies. J Nanomed Nanotechnol 7: 415. doi: 10.4172/21577439.1000415

Page 6 of 8

\section{Effect of Anti-LD Antibody on Contraction Characteristics and MgATPase Activity in Single Skinned Fibers}

We also examined the effect of monoclonal antibody (anti-LD antibody) to two light chains in myosin head lever arm domain, located at the proximal region of myosin head (Figure 14) [23]. As has been the case in the effect of anti-S-2 antibody, anti-LD antibody (up to $1.5 \mathrm{mg} / \mathrm{ml}$ ) decreased the $\mathrm{Ca}^{2+}$-activated isometric force in skinned muscle fibers in a time and dose dependent manner, without appreciably changing its MgATPase activity (Figure 15). Despite the marked reduction of $\mathrm{Ca}^{2+}$-activated isometric force, the value of Vmax remained unchanged as can be seen in Figure 16, in which P-V curves (Figure 16A) are also shown to be identical when normalized with respect to the maximum isometric force (Figure 16B). Muscle fiber stiffness, on the other hand, decreased in proportion to isometric force, again indicating that myosin heads, with anti-LD antibodies attached to their LD region, hydrolyze ATP at the rate similar to that of native heads, while they no longer generate isometric force without being sensed by small length perturbations as stiffness and without producing appreciable internal resistance against fiber shortening [23].

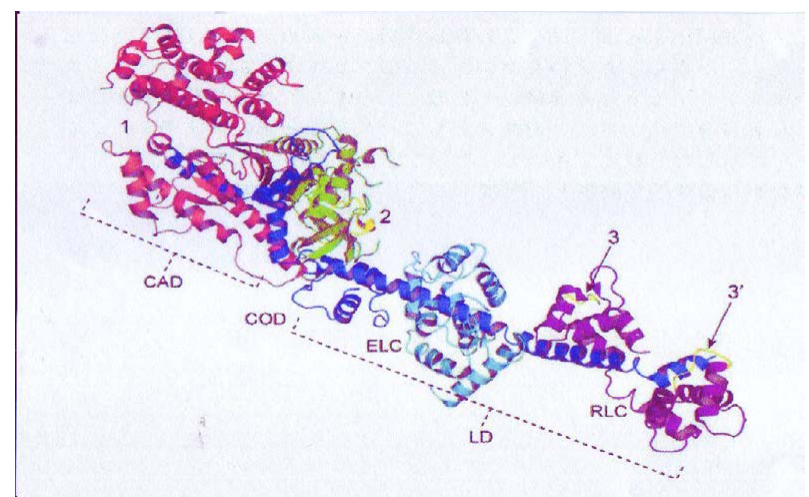

Figure 14: Structure of myosin head, consisting of CAD, COD, and LD domains. The region of attachment of anti-LD antibody to two light chains in LD is indicated by numbers 3 and 3 ' [9].

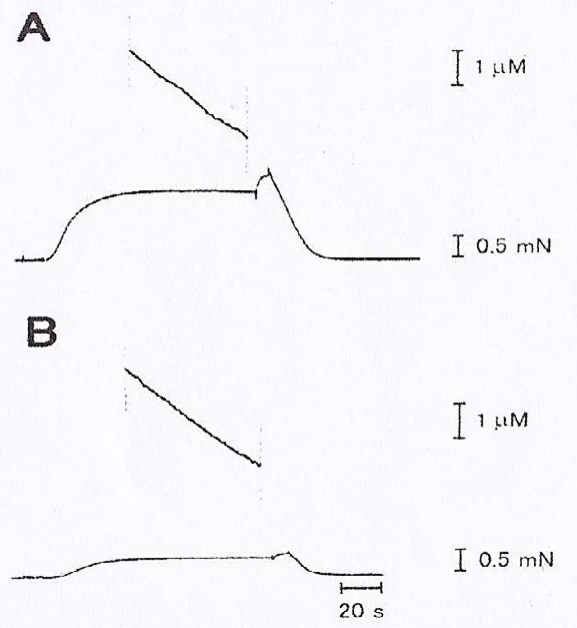

Figure 15: Simultaneous recordings of MgATPase activity (upper traces) and $\mathrm{Ca}^{2+}$-activated isometric force (lower traces) in skinned muscle fibers before (A) and after application of anti-LD antibody $(2 \mathrm{mg} / \mathrm{ml})$ [22].
The puzzling effect of both anti-S-2 and anti-LD antibodies on contraction characteristics and MgATPase activity can readily be accounted for by assuming that myosin heads with antibodies attached either to their LD or S-2 region repeat attachment-detachment cycle with actin filaments without formation of strong A-MADP binding and A-M binding, so that they hydrolyse ATP at the normal rate, but are readily detached from actin filaments by small length perturbations and by muscle fiber shortening. This explanation rests on the assumption that (1) contrary to the dogma, weak to strong A-M binding transition is not necessarily coupled with actin-activated myosin ATPase activity, and that (2) antibodies attached to LD or S-2 region can affect the strength of A-M binding at the myosin head-actin filament interface, distant from both LD and S-2 regions. The possible function of LD and $\mathrm{S}-2$ regions in regulating the strength of A-M binding is supported by our experiments using the centrifuge microscope, as described below.

\section{Effect of Anti-S-2 Antibody on Strength of Binding between Myosin Heads and Actin Filaments as Revealed by Experiments Using Centrifuge Microscope}

Figure 17 illustrates the centrifuge microscope system developed by us in 1990 [24]. Polystyrene beads (diameter, 4.5 or $2.8 \mu \mathrm{m}$; specific gravity, 1.3), coated with rabbit skeletal muscle myosin, are made to slide in the presence of ATP along well-organized actin filament arrays in giant intermodal cell of green algae, mounted in a cuvette on the rotor of the centrifuge microscope. Myosin-coated beads move along actin cables under various centrifugal forces, serving as external loads, can be observed and video-recorded with a stroboscopic device. Without application of centrifugal forces, the steady unloaded velocity of bead movement, corresponding to the maximum unloaded shortening velocity Vmax in muscle fibers, was 1.6-3.6 $\mu \mathrm{m} / \mathrm{s}$ in both beads coated with control myosin and beads coated with anti-S-2 antibody-treated (up to $0.3 \mathrm{mg} / \mathrm{ml}$ ) myosin [24] (Figure 18). The similarity of maximum unloaded sliding velocity between beads coated with control myosin and those coated with antibody-treated myosin at low antibody concentrations may be comparable to the similarity of Vmax between control and antibody-treated muscle fibers (Figures 13 and 16). However, the centrifugal force required to detach moving beads was found to be much smaller in the antibody-treated myosin than in the
A

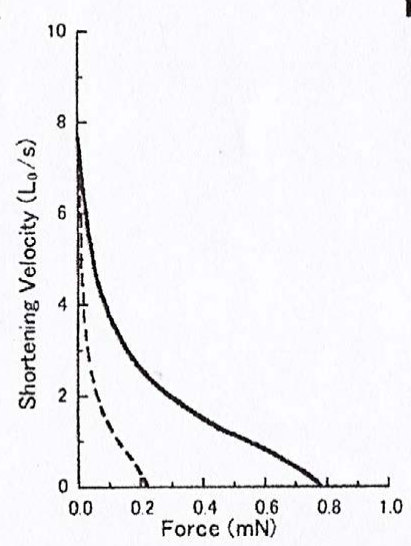

B

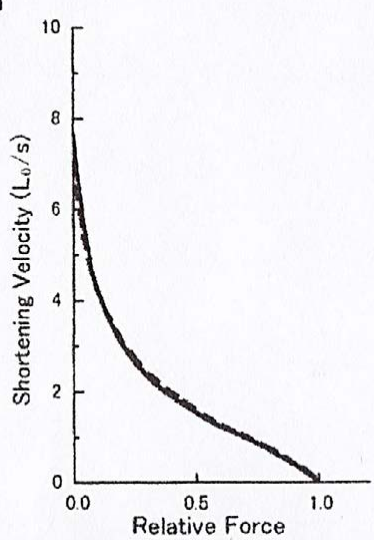

Figure 16: Effect of anti-LD antibody on force-velocity relation of single skinned muscle fiber. (A) Force-velocity curves before (solid line) and after application (broken line) of anti-LD antibody $(1.5 \mathrm{mg} / \mathrm{ml})$. (B) The same force-velocity curves normalized with respect to maximum isometric forces [22]. 
Citation: Sugi H, Chaen S (2016) Evidence for the Regulation of Actin-myosin Binding Strength by Lever Arm and Subfragment-2 Regions of Myosin Molecule in Contracting Skinned Muscle Fibers as Revealed by the Effect of Antibodies. J Nanomed Nanotechnol 7: 415. doi: 10.4172/21577439.1000415

Page 7 of 8

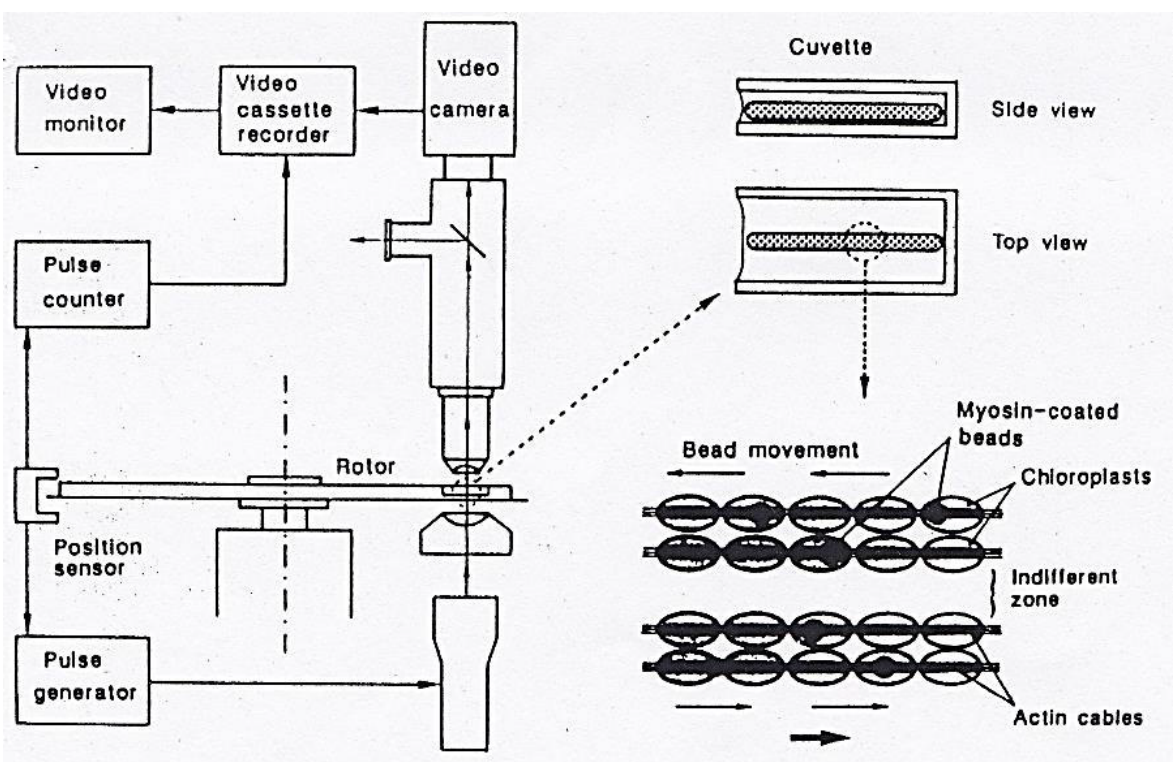

Figure 17: Diagram illustrating the centrifuge microscope system to record ATP-dependent sliding movement of polystyrene beads, coated with rabbit skeletal muscle myosin, along well organized actin filament arrays in giant algal cells, mounted on the rotor of centrifuge microscope. The bead movement is recorded stroboscopically [23].

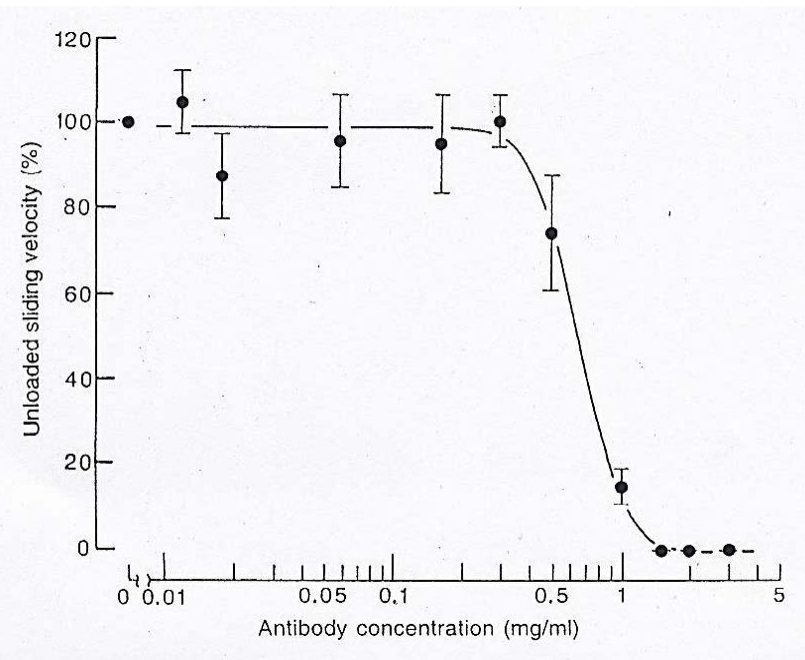

Figure 18: Effect of anti-S-2 antibody on the steady unloaded skidding velocity of myosin-coated beads along actin cables [24].

control myosin, being $0.5-3.5 \mathrm{pN}(2.5 \pm 1.1 \mathrm{pN}$, mean $\pm \mathrm{SD}, \mathrm{n}=8)$ in the former, and $7.5-18.0 \mathrm{pN}(9.1 \pm 4.0 \mathrm{pN}$, mean $\pm \mathrm{SD}, \mathrm{n}=10)$ in the latter [25]. This result clearly indicates that the strength of binding at myosinactin filament interface is markedly reduced by anti-S- 2 antibody.

\section{Conclusion}

In our previous review article [25], we presented evidence against the swinging lever arm mechanism, which is cited in many text books as an established dogma in muscle contraction. In this article, we have presented experimental evidence for the essential role of myosin head lever arm domain (LD) and myosin subfragment-2 region (S-2) in producing muscle contraction, by showing that myosin molecules with either anti-LD or anti-S-2 antibodies attached can no longer generate isometric force in skinned muscle fibers, while they hydrolyse ATP at a rate similar to that in native myosin molecules [22,23]. A puzzling problem, that arises with this result is, why such myosin heads cannot be sensed by small length perturbations as muscle fiber stiffness and produce no appreciable internal resistance against shortening. The only way to explain the puzzling phenomena is that ATP hydrolysis coupled with cyclic attachment-detachment between myosin heads and actin filaments is not necessarily require transition from weak to strong binding of myosin heads with actin filaments; in other words, ATP hydrolysis may go on without passing through strong binding between myosin heads and actin filaments, contrary to the generally accepted view of weak to strong binding transition coupled with ATP hydrolysis. This implies that the difference in strength of A-M binding between A-M-ADP-Pi and A-M-ADP, determined by equilibrium dissociation constant in solution may not apply for the A-M binding in the 3D myofilament lattice in muscle. Much more experimental work is necessary to solve this issue; muscle is still filled with unsolved mysteries.

\section{Acknowledgements}

We dedicate this article to the late Professor Takakazu Kobayashi, who constructed ingenious device to obtain the whole range of $P-V$ curve in skinned fibers in one shot, and suddenly passed away in July this year. By the way, we thank Aurora Scientific Inc, Aurora, Canada for providing length and force control apparatus, with which we could perform experiments described in this article.

\section{References}

1. Sugi H (1992) Muscle Contraction and Cell Motility: Molecular and Cellular Aspects. Berlin: Springer.

2. Bagshaw CR (1993) Muscle Contraction. London: Chapman and Hall.

3. Huxley HE, Hanson J (1954) Changes in the cross striations of muscle during contraction and stretch. Nature 173: 973-976.

4. Page SG, Huxley HE (1963) Filament lengths in striated muscle. J Cell Biol 19: $369-390$.

5. Huxley HE (1969) The mechanism of muscular contraction. Science 164: 1358 1366.

6. Sugi H, Akimoto T, Sutoh K, Chaen S, Oishi N, et al. (1997) Dynamic electron 
Citation: Sugi H, Chaen S (2016) Evidence for the Regulation of Actin-myosin Binding Strength by Lever Arm and Subfragment-2 Regions of Myosin Molecule in Contracting Skinned Muscle Fibers as Revealed by the Effect of Antibodies. J Nanomed Nanotechnol 7: 415. doi: 10.4172/21577439.1000415

Page 8 of 8

microscopy of ATP-induced myosin head movement in living muscle thick filamenrs. Proc Natl Acad Sci 94: 4378-4382.

7. Sugi $H$, Minoda $H$, Inayoshi $Y$, Yumoto F, Miyakawa $T$, et al. (2008) Direct demonstration of the cross-bridge recovery stroke in muscle thick filaments in aqueous solution by using the hydration chamber. Proc Natl Acad Sci USA 105: 17396-17401.

8. Sugi H, Chaen S, Akimoto T, Minoda H, Miyakawa T, et al. (2015) Electron microscopic recording of myosin head power stroke in hydrated myosin filaments. Sci Rep 5: 15700.

9. Minoda H, Okabe T, Inayoshi Y, Miyakawa T, Miyauchi Y, et al. (2011) Electron microscopic evidence for the myosin head lever arm mechanism in hydrated myosin filaments using the gas environmental chamber. Biochem Biophys Res Commun 405: 651-656.

10. Lymn RW, Taylor EW (1971) Mechanism of adenosine triphosphatase hydrolysis by actomyosin. Biochemistry 16: 4617-4624.

11. Cecchi G, Griffiths PJ, Taylor SW (1984) The Kinetics of cross-bridge attachment and detachment studied by high frequency stiffness measurements. In Contractile Mechanisms in Muscle, Pollack GH, Sugi H (eds), New York: Plenum 641-655.

12. Julian FJ, Sollins MR (1975) Variation of muscle stiffness with force at increasing speeds of shortening. J Gen Physiol 66: 287-302.

13. Huxley AF (1957) Muscle structure and theories of contraction. Prog Biophys Biophys Chem 7: 255-318.

14. Suzuki S, Sugi $H$ (1983) Extensibility of the myofilaments in vertebrae skeletal muscle as revealed by stretching rigor muscle fibers. J Gen Physiol 81: 531-546.

15. Huxley HE, Stewart A, Sosa H, Irving T (1994) X-ray diffraction measurements of the extensibility of actin and myosin filaments in contracting muscle. Biophys J 67: 2411-2421.

16. Reisler $S$ (1980) on the question of co-operative interaction of myosin heads with F-actin in the presence of ATP. J Mol Biol 138: 93-107.
17. 17. Knight $P$, Offer $G$ (1980) Investigation, by cross-linking, of conformational changes in F-actin during its interactions with myosin. Biochemistry 19: 4682-4687.

18. Chaen S, Shimada M, Sugi H (1986) Evidence for cooperative interactions of myosin heads with thin filament in the force generation of vertebrate skeletal muscle fibers. J Biol Chem 261: 13632-13636.

19. Tokiwa T, Morales MF (1971) Independent and cooperative reactions of myosin heads with F-actin in the presence of adenosine triphosphate. Biochemistry 10 1722-1727.

20. Edman KAP (1979) The velocity of unloaded shortening and its relation to sarcomere length and isometric force in vertebrate muscle fibres. J Physio 291: 143-159.

21. Brenner B (1998) Muscle Mechanics II: Skinned muscle fibres. In: Sugi H (ed.) "Current Methods in Muscle Physiology", Oxford: Oxford University Press, 33-69.

22. Sugi H, Kobayashi T, Gross T, Noguchi K, Karr T (1992) Contraction characteristics and ATPase activity of skeletal muscle fibers in the presence of antibody to myosin subfragment-2. Proc Natl Acad Sci USA 89: 6134-6137.

23. Sugi H, Chaen S, Kobayashi T, Abe T, Kimura K, S et al. (2014) Definite differences btween in viro actin-myosin sliding and muscle contraction as revealed using antibodies to myosin head. PLOS ONE 9: e93272.

24. Oiwa K, Chaen S, Kamitsubo E, Shinmen T, Sugi H (1990) Steady-state forcevelocity relation in the ATP-dependent sliding movement of myosin-coated beads on actin cables in vitro studied using a centrifuge microscope. Proc Natl Acad Sci USA 87: 7893-7897.

25. Tsuchiya T, Tanaka H, Shirakawa I, Karr T, Sug H (1998) Evidence for the essential role of myosin subfragment-2 in the ATP-dependent acin-myosin sliding in muscle contraction. Jpn J Physiol 48: 383-387.

26. Sugi $H$, Chaen $S$ (2016) Evidence against the swinging lever arm mechanism in muscle contraction based on the effect of antibodies to myosin hea. J Nanomed Nanotechnol 7: 1000377. 